\title{
Online Impedance Estimation of Sealed Lead Acid \& Lithium Nickel - Cobalt - Manganese Oxide Batteries using a Rapid Excitation Signal
}

This paper was downloaded from TechRxiv (https://www.techrxiv.org).

\section{LICENSE}

CC BY 4.0

SUBMISSION DATE / POSTED DATE

$07-01-2020 / 17-07-2020$

\section{CITATION}

Alao, Olakunle; Barendse, Paul (2020): Online Impedance Estimation of Sealed Lead Acid \& Lithium Nickel Cobalt - Manganese Oxide Batteries using a Rapid Excitation Signal. TechRxiv. Preprint. https://doi.org/10.36227/techrxiv.11536281.v2

$\mathrm{DOI}$ 


\title{
Online Impedance Estimation of Sealed Lead Acid \& Lithium Nickel-Cobalt-Manganese Oxide Batteries using a Rapid Excitation Signal
}

\author{
Olakunle Alao, Student Member, IEEE, and Paul Barendse, Member, IEEE
}

\begin{abstract}
Electrochemical Impedance Spectroscopy (EIS) has gained traction as a technique apt for condition monitoring of batteries. The drawback of EIS is that it is only applicable when the system is offline (i.e. it must be disconnected from the load), takes a long time to complete and requires an expensive equipment for measurement. This work aims to adapt the EIS to serve as an in-situ measurement technique, that can be utilized for online condition monitoring of two unique battery chemistries - lead acid and lithium NCM. This work develops in twofold - firstly, the Chirp broadband signal is proposed amongst a variety of other broadband signals to significantly shorten the time required for EIS measurement. Subsequently, a power converter that is typically used to interface a battery with the load for current and voltage regulation functions, is utilized for online condition monitoring of both batteries through closed loop control of the power converter and duty-cycle perturbation. This combined approach presents a novel low-cost technique for online condition monitoring of batteries, with the ability to complete battery characterization in a very short time. In this regard, EIS measurement is completed for a lead acid battery (with lowest EIS characterization frequency of $0.1 \mathrm{~Hz}$ ) in 5 seconds and lithium NCM battery (with lowest EIS characterization frequency of $20 \mathrm{mHz}$ ) in 25 seconds.
\end{abstract}

Index Terms - EIS, Broadband Excitation, DC-DC Converter, Sealed Lead Acid, Lithium Nickel-Cobalt-Manganese Oxide

\section{INTRODUCTION}

$\mathrm{T}$ HERE is an insatiable demand for energy in modern society, with more applications tending towards grid independence and mobility. Previously, the energy demand has been met by fossil fuel technologies. The increasingly diminutive nature of fossil fuel and greenhouse gas emissions has prompted the need for clean energy sources. Renewable energy sources such as solar and wind are experiencing rapid growth, with most new investments in the global electricity sector focused towards their development. Due to the variability of renewable energy systems, energy storage systems (ESS) must thus be readily available to ensure efficient and optimal operation [1]. ESS development is thus crucial if renewable energy technologies are to phase-out fossil fuel technologies.

Manuscript received XXXXXXX XX, XXXX; revised XXXXXXX XX, XXXX; XXXXXXX XX, XXXX. Date of publication XXXXXXX XX, XXXX. "This research is supported by the Advanced Machines and Energy Systems (AMES) Group, Dutkiewicz Family and Postgraduate Bursary of the University of Cape Town."

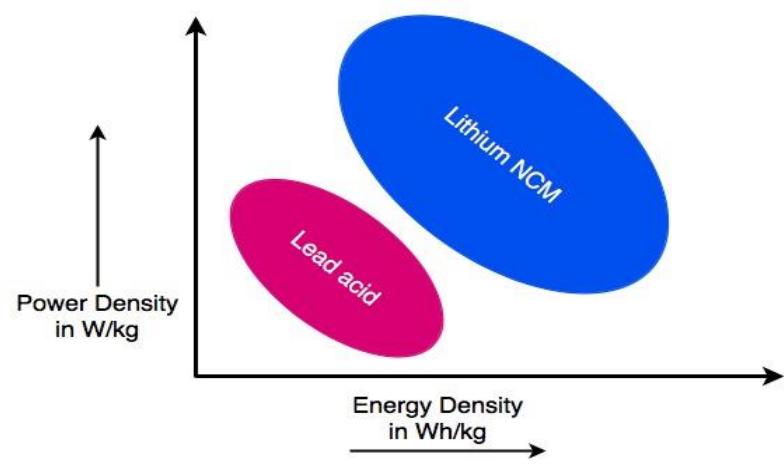

Fig.1 Comparison of lead acid and lithium NCM against power and energy density

Batteries are utilized in a range of applications such as: consumer electronics, electrified transportation and renewable energy storage systems. On one hand, lead acid batteries - one of the oldest battery chemistries, are still largely applied in renewable energy systems - particularly in grid energy storage systems, because of their affordability, reliability and recyclability [1] - [3]. On the other hand, lithium ion batteries due to their high energy and power density (see Fig. 1), long service life and low self-discharge rate are widely utilized in the electrified transportation and consumer electronics industries [4]. Since batteries are utilized in systems that require a high level of reliability, fidelity and safety; a battery management system (BMS) is therefore required for condition monitoring of batteries, to increase safety and life expectancy. Electrochemical Impedance Spectroscopy (EIS) has most recently been widely used for characterization of batteries, because of its robustness and capability to estimate very slow dynamics of the battery as opposed to the internal DC resistance, Coulomb counting, electrolyte concentration, stepresponse, and harmonic small-signal system excitation techniques etc. [4] - [6].

Notwithstanding the robustness of the classical EIS for battery characterization, its severely constrained by measurement time, because its measurements are carried out

Olakunle Alao and Paul Barendse are with the Department of Electrical Engineering, Advanced Machines and Energy Systems Group, University of Cape Town, Cape Town 7701, South Africa (e-mail: alxola001@myuct.ac.za; paul.barendse@uct.ac.za). 
using single sine (of a given frequency) perturbation, with a wait time $t$ between each user-selected frequency interval. This takes a considerable amount of time - in the order of minutes, making it unsuitable for online condition monitoring [3] - [6].

Another important aspect of EIS is the validity of measurement. For EIS measurements to be deemed valid, it should adhere to Kramers-Kronig relations of system linearity, stability, and causality. Since batteries are inherently nonlinear systems as propounded by Butler-Volmer's equation [8] - [9]; thus, to observe the linearity and causality conditions of Kramers-Kronig, the perturbation amplitude must be small enough to allow the system to be measured at a quasi-linear point of its polarization curve [7]. A small perturbation amplitude corresponds to a low signal-to-noise ratio (SNR); whereas, a high perturbation amplitude corresponds to a high SNR - alongside time domain distortions, appearing as harmonics in the frequency domain [8] - [9]. It is therefore important that the EIS measurement is optimized to obtain an optimal value compromise between achieving a high SNR and obeying the linearity and causality conditions.

This paper thus aims to adapt the EIS to serve as an in-situ measurement technique, that can be utilized for online condition monitoring of two different battery chemistries whilst adhering to the Kramers-Kronig relation. In this regard, a novel low-cost online condition monitoring technique is proposed for characterization of both batteries; thus, eliminating the need to disconnect the batteries from the system in operation and significantly reducing measurement time from the order of minutes to few seconds. In this paper, this time reduction corresponds to 5 seconds for the lead acid battery (with a time constant of 10 seconds) and 25 seconds for a lithium NCM battery (with a time constant of 50 seconds). The remainder of this paper is organized as follows: Section II describes the methodology for battery characterization using the EIS technique. Section III discusses the time-frequency analysis of broadband signals. Section IV discusses online condition monitoring systems in literature and presents the proposed online condition monitoring system utilized in this paper. Section V briefly describes the hardware setup of the FRA and the low-cost system. Section VI presents the results and Section VII concludes the paper.

\section{ELECTROCHEMICAL IMPEDANCE SPECTROSCOPY}

Electrochemical Impedance Spectroscopy (EIS) is a valuable electro-analytic technique used to investigate electrochemical systems (batteries) under different conditions. EIS is undertaken by injecting a sinusoidal excitation signal (potential or current) on the battery's operating point; and observing the associated response (current or potential) of the battery. In the galvanostatic mode, an alternating current is injected, and the associated voltage response is measured; whereas, in the potentiostatic mode, an alternating voltage is applied, and the associated current response is measured [7]. In this paper, EIS measurement is undertaken using the galvanostatic mode, since batteries are low-impedance device and an application of a $10 \mathrm{mV}$ (potentiostatic mode voltage) sine wave produces amperes of current-flow, resulting in the evolution of the system and a corresponding subtle change in SoC during the measurement period [5] - [7]. The perturbation and response-

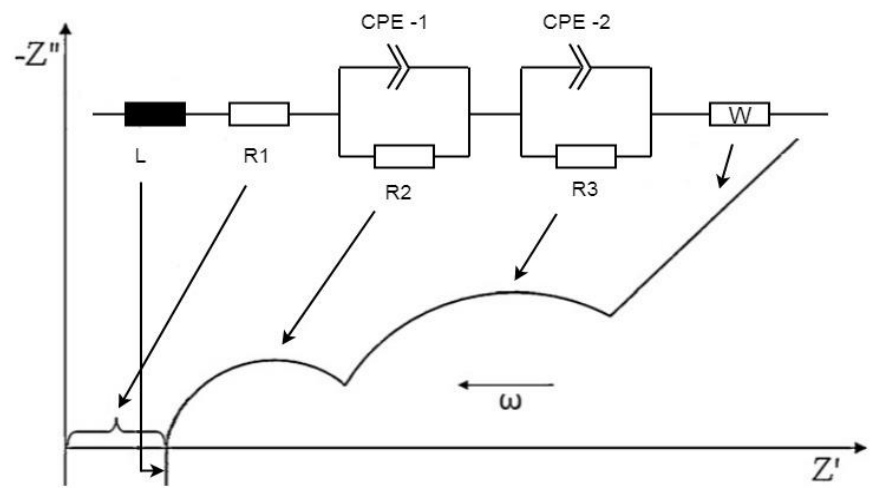

Fig. 2 Equivalent circuit often used for description of the electrochemical processes and their corresponding Nyquist shape
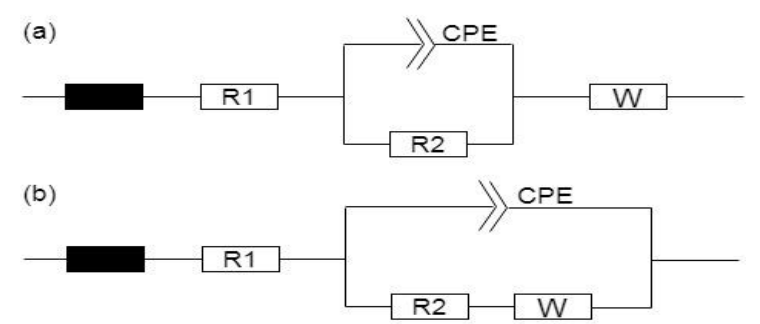

Fig. 3 Randles Circuit of (a) Lithium NCM battery and (b) Lead acid battery

signal of the galvanostatic EIS is presented in (1) and (2). The input signal and response signal - with a phase shift, are analyzed as a sum of sinusoidal functions. This procedure is repeated for several user-defined frequencies of interest of the perturbation signal [7]. Subsequently, the complex ohms law relationship is used to compute the impedance as (3). In (3), $I_{\max }$ and $V_{\max }$ are the amplitudes of the current and voltage signal respectively, $f$ is the frequency and $\phi$ is the phase shift.

$$
\begin{gathered}
\Delta I=I_{\text {max }}(\sin 2 \pi f t) \\
\Delta V=V_{\text {max }}(\sin 2 \pi f t+\phi) \\
Z=\frac{V_{\text {max }}(\sin 2 \pi f t+\phi)}{I_{\max }(\sin 2 \pi f t)}
\end{gathered}
$$

The Nyquist plot as Fig. 2 represents the separated form of the real and imaginary part of the impedance. The imaginary part is on the vertical axis and the real part is on the horizontal axis. The Nyquist plot is fitted to the equivalent circuit models in Fig. 3, that relates the associated physicochemical process occurring in the battery using the complex nonlinear leastsquares (CNLS).

The impedance of a battery is composed of inductive reactance, double layer capacitance, charge transfer and Warburg impedance. The high frequency region of the Nyquist plot corresponds to the inductive reactance - which is due to the metals in the measurement setup [5] - [7]. The ohmic resistance is the high frequency intercept of the plot at the real axis and is due to the resistance of the electrode, electrolyte and separators. The semicircle corresponds to the charge transfer resistance and the capacitance at the electrode-electrolyte double layer. The low frequency $\left(45^{\circ}\right)$ line relates to the diffusion process. [5] [7]. Since these parameters vary with the state of the battery, they must thus be accurately derived and monitored. 


\section{BROADBAND EXCITATION SIGNALS}

EIS measurements suffers from a long measurement time. Broadband excitation signals such as: Multisine, Chirp and Pseudo-Random Binary Sequence (PRBS) presents a more rapid approach to EIS measurement. However, the increase in measurement speed that broadband signal offers, results in an accompanying compromise of the impedance spectrum accuracy. Broadband signals have been widely applied in the bio-medical field [10] - [12]. In these applications, a high measurement Signal to Noise Ratio (SNR) is necessary to derive meaningful tissue measurements. More so, these measurements typically involve a high magnitude excitation signals - with a large crest factor (CF). Notwithstanding, in any application, the adherence to the linearity and time invariance conditions are critical. The different broadband signals have unique frequency and time properties; hence, the $\mathrm{CF}$ is an indicator for comparing these signals [10] - [11]. The CF of an excitation signal $-u[n]$ is defined as the ratio of its maximum value $l_{\max }$ to its root mean square value $l_{\text {max }}[11]$ as (7):

$$
C F(u)=\frac{l_{\max }}{l_{r m s}}=\frac{\underset{n \in[0, N-1]}{\max } \mid u[n]}{\sqrt{\frac{1}{N} \sum_{n=0}^{N-1}|u[n]|}}
$$

$\mathrm{CF}$ is a measure of how much amplitude the signal consumes to inject a certain power level into the system. CF relates to the signal's compactness. The lower the CF, the more energy is transferred to the system, resulting in a better signal-to-noise ratio. The $\mathrm{CF}$ ranges from 1 - the ideal to infinity - the worst possible case. The reduction of $\mathrm{CF}$ will permit a larger energy to be injected into the system. It will also ensure that the system is operating in the linear region [10] - [12].

As earlier asserted, many broadband signals exist. The multisine signal involves the summing of sinusoids of userdefined frequencies of interest. This significantly reduces the measurement time, albeit limited by the lowest excited frequency as in [12] - [13]. Also, it suffers from a high CF due to an increased amplitude. PRBS was selected as the signal of choice for electrochemical system characterization in [14] [15]. Its drawback is that it is only able to obtain a low (or socalled 'unitary $\mathrm{CF}^{\prime}$ ') when the full frequency band is utilized by the user, which is not plausible in battery applications, since only a range of frequencies are required to characterize the battery [14] - [15]. This ultimately results in a loss of accuracy. The chirp signal has a CF of 1.4 and can complete rapid EIS measurements. The chirp broadband signal has been proposed for electrochemical systems in [16] - [17] but is yet to be applied physically to batteries.

\section{A. Multisine Signal}

A real-valued multisine as Fig. 4(a), the time-domain expression is a Fourier series of order $N$ as (8)

$$
u(t)=\sum_{n=0}^{N-1} a_{n} \cos \left(2 \pi f_{n} t+\varphi_{n}\right)
$$

In (8), $N$ is the number of user-selected excitation frequencies, $a_{n}$ is the amplitude matrix, $f_{n}$ is the excited frequencies, and $\varphi_{n}$ is the phase matrix. The multisine signal's rms is independent of the phases $(\varphi)$ but is dependent on the amplitude $\left(a_{n}\right)$ and number of signals considered. The maximal peak can be significantly compressed depending on the choice of the selected frequency's phase vectors, which-

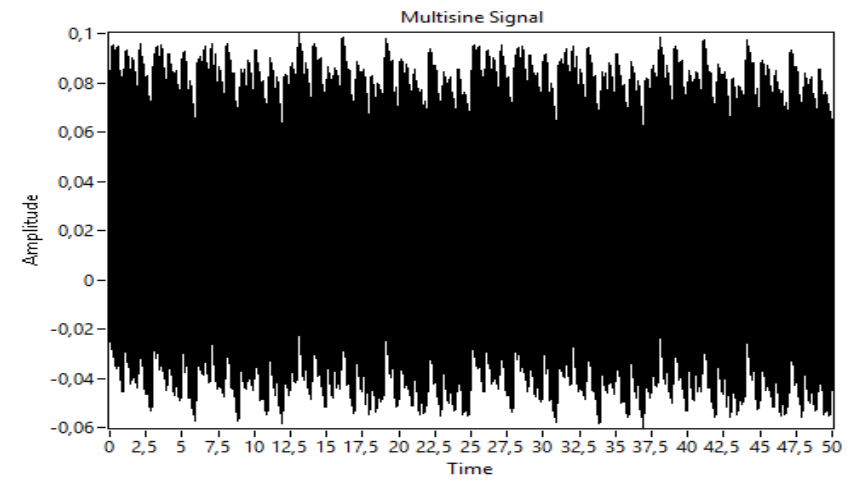

Fig. 4(a) Optimized Multisine Signal with $\mathrm{CF}=2.86$

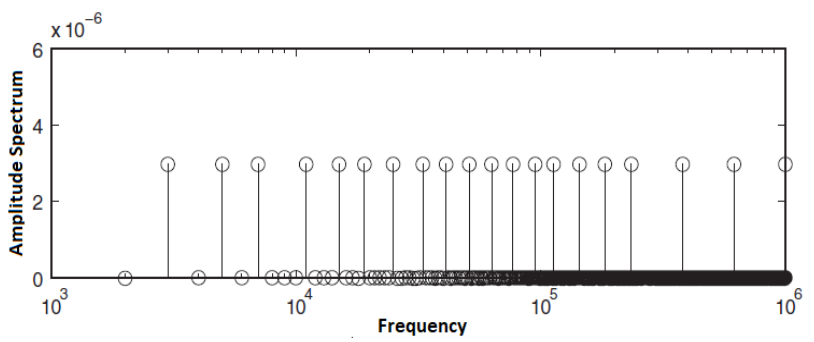

Fig. 4(b) Frequency spectrum of the multisine signal [10]

results in the minimization of the $\mathrm{CF}$. The Multisine has a flat frequency spectrum as shown in Fig. 4(b), and its excitation energy is evenly distributed over the user-defined frequencies. It also has a high peak-peak magnitude (CF); thus, making it crucial to carefully optimize: frequency, amplitude and phase to reduce $\mathrm{CF}$ as in [6], [11], [15].

\section{(a) Frequency Optimization}

The frequency is distributed to minimize measurement time and maximize resolution of the impedance spectrum. A small number of excited frequencies that are evenly distributed will provide similar information to that of the logarithmic spacing; however, the disadvantage of evenly distributed frequencies, is the high number of excitation frequency required for robust system characterization [6], [11], [15]. Likewise, evenly distributed frequencies suffer from harmonics at the fundamental frequency of the excitation signal. To avoid the presence of harmonic frequencies that results from even nonlinearities, a log-odd frequency distribution is applied as proposed in [9] and shown in (9).

$$
f_{\mathrm{n}}= \begin{cases}\left(f_{o} \times 2^{n}\right) & f_{n}<1 \\ \left(f_{o} \times 2^{n}\right)+1 & f_{n} \geq 1\end{cases}
$$

\section{(b) Amplitude Optimization}

The summing of sinusoids - which is required for robust system characterization, increases the signal's maximal peak. Nonetheless, for (quasi-) linearity to be assumed, it is paramount that the maximum peak is small. The amplitude is thus optimized to obtain appropriate amplitudes of the harmonic spectrum, resulting in a maximization of the signal energy at the user-defined frequencies of interest. The multisine amplitude is designed from the observation of the Bode plot of the batteries. The signal amplitude is thus selected to follow an inverse pattern of the Bode plot. Amplitude optimization reduces $\mathrm{CF}$ and maximizes signal energy. 


\section{(c) Phase Optimization}

The phase optimization minimizes the maximal peak in the time domain, so that the excitation frequencies' power level is maintained. A low $\mathrm{CF}$ can be attained by simply varying the phase difference from 0 to 360 degrees $(0-2 \pi)$ between the consecutively adjacent frequencies. Nonetheless, the phases of the multisine is optimized in this paper to decrease the maximum peak in the time domain through $\mathrm{CF}$ and non-linear optimization. The multisine phases are optimized using the Newman's phases as (10):

$$
\phi_{n}=\frac{\pi n^{2}}{N}, n=0: N-1
$$

\section{B. Pseudo-random binary sequence signal}

The Pseudo-Random Binary Sequence (PRBS) signal as in Fig. 5, is a periodic signal that possesses white-noise-like properties [14] - [15]. PRBS characterizes linear systems within a range of frequencies and can be generated through linear feedback shift registers (LFSR) or a combination of XOR Logical Operator and Unit Delay. The PRBS's power is not all concentrated at the impedance frequency band of interest; thus, for implementing a flat spectrum, only a minor portion of the energy is utilized. The Fourier transform of the resulting impedance from the time domain voltage and current signal in Fig. 5, can be utilized to determine the usable range of frequencies. The usable range of frequencies is defined by the half-power bandwidth ( $3 \mathrm{~dB}$ bandwidth) and is indicated in the frequency response in Fig. 6 .

\section{(a) Selection of PRBS length}

An increase in bit-length results in an increase in the number of states, usable bandwidth and test time [14] - [15]. Therefore, for the design of a PRBS signal, the following parameters must be considered: the frequency of interest, usable bandwidth and test time. For a PRBS with $n$-bit (polynomial - order of shift register), the number of possible-states $N$ is mathematically expressed as (11). The order $n$, alongside the delay time interval $T_{c}$ defines the maximum period length $T_{\max }$ of the PRBS as (12). The power spectrum of a periodic PRBS signal with a delay time interval $T_{c}$ is expressed as (13).

$$
\begin{gathered}
N=2^{n}-1 \\
T_{\max }=\left(2^{n}-1\right) \times T_{c}=\mathrm{N} \times T_{c} \\
\phi^{p}\left(m \frac{2 \pi}{N T_{c}}\right)=\frac{1}{N} \phi^{d}(\mathrm{~m})\left|\frac{\sin \frac{m \pi}{N}}{\frac{m \pi}{N}}\right|^{2}
\end{gathered}
$$

(b) Selection of PRBS clock frequency

Another important parameter for PRBS design is the clock pulse frequency $f_{c}$, which is the register's shift frequency. The parameters that determine the usable frequency range of a PRBS are the number of bits and the clock pulse frequency [15]. Thus, the minimum and maximum frequency are expressed as (14). A PRBS signal's data is embedded in certain points of the frequency band, termed frequency step and corresponds to the minimum frequency and is expressed as (15).

$$
\begin{gathered}
f_{\text {min }}=\frac{f_{c}}{N} ; f_{\text {max }}=\frac{f_{c}}{3} \\
f_{\text {step }}=f_{\text {min }} ; f_{\text {min }}: f_{\text {step }}: f_{\text {max }}
\end{gathered}
$$

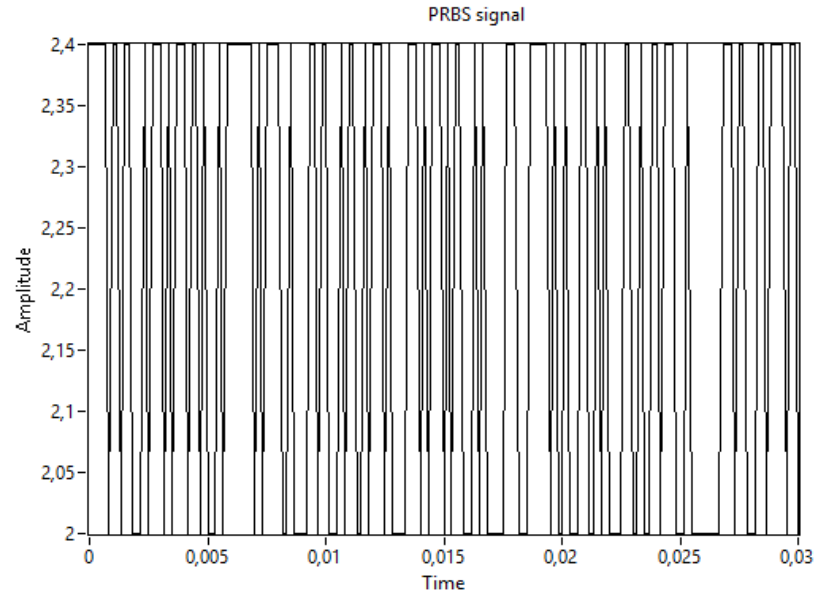

Fig. 5 Time-domain PRBS signal with $\mathrm{CF}=1.78$

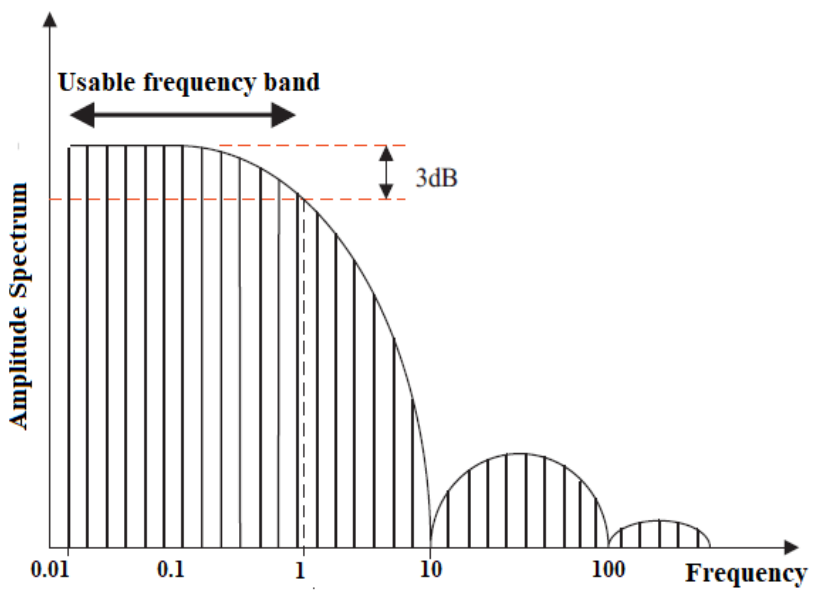

Fig. 6 Power spectrum of a PRBS indicating usable frequency band [15].

Table 1. PRBS time-length optimization

\begin{tabular}{lcc}
\hline \hline PRBS Parameters & Lithium NCM & Lead Acid \\
\hline \hline Frequency Range 1 $(\mathrm{Hz})$ & $0.02: 0.02: 3.4$, & $0.1: 0.1: 8.5$, \\
Frequency Range 2 $(\mathrm{Hz})$ & $5.865: 5.865: 2000$ & $17.6: 17.6: 6000$ \\
Clock Frequency $(\mathrm{Hz})$ & $10.22,6000$ & $25.5,18000$ \\
PRBS Bits - $\boldsymbol{n}$ & 9,10 & 8,10 \\
Testing Time $(\mathrm{sec})$ & $50,0.17$ & $10,0.056$ \\
Total Testing Time $(\mathbf{s e c})$ & 50.17 & 10.056 \\
\hline
\end{tabular}

\section{(c) PRBS optimization}

As the bit length increases, the bandwidth increases, and the test time increases, both exponentially. Thus, the chosen bit length should be selected in such a way that it falls within the EIS range within the shortest test time (see Table 1). From EIS study on both batteries (see [5]), it has been deduced that the critical frequency band that robustly characterizes the lead acid and lithium ion batteries are $(5 \mathrm{kHz}-100 \mathrm{mHz})$ and $(2 \mathrm{kHz}-$ $20 \mathrm{mHz}$ ) respectively. Since both frequency bands span over four decades, the PRBS for both batteries were designed with two wave forms of different clock frequencies, to ensure that the bands are sufficiently covered (see Table 1). 


\section{Chirp signal}

Chirp signals as in Fig. 5(a) have been vastly applied in the sonar and radar field, albeit sparingly applied in electrochemistry. Reference [16] - [17]; and most recently [6] and [18] verified the selection of chirp as the signal of choice for electrochemical system characterization. Depending on the kind of frequency variation, the frequency of a chirp signal can ramp up or down with time [16] - [17]. The chirp time-domain function is expressed in (16) and the chirp rate in (17):

$$
\begin{gathered}
c(t)=A \sin (2 \pi f(t) t)) \\
f(t)=f_{\min }+\left(\frac{f_{\max }-f_{\min }}{2 T}\right) t
\end{gathered}
$$

In (16) and (17), $A$ is the amplitude, $t$ is the simulation time, $f_{\min }$ is the initial frequency and $T$ is the target time. The advantage of the chirp signal is that it can be generated in a very short time and has a large bandwidth. From Fig. 5(b), it can be deduced that the signal energy is concentrated at the high frequencies. Thus, the drawback of the chirp signal is that the impedance information at the low frequency region is scattered because of a poor SNR. Unlike the multisine and PRBS that are converted to the frequency domain using the Fast Fourier Transform (FFT), the chirp is processed using the Short-time Fourier transform (STFT); because of its time-varying frequency nature.

\section{(a) Short-time Fourier Transform (STFT)}

The STFT utilizes the Discrete Fourier Transform (DFT) to analyze the properties of a time-varying frequency signal. In a chirp (time-varying frequency) signal, time optimization reduces the resolution of frequency; and frequency optimization reduces the resolution of time. STFT is derived by partitioning a long time-domain signal into several time windows of equal length. These windows overlap each other to decrease the artefacts at the boundary. Subsequently, Fourier Transform is applied separately on each separate window [19]. The STFT of a signal is expressed as (18):

$$
X[m, k]=\sum_{n=0}^{L-1} x[m+n] e^{-j \frac{2 \pi}{L} n k}
$$

In (18), $m$ is the starting point for the localized DFT, $k$ is the DFT's index at that point and $[m+n]$ is the signal samples. Likewise, the STFT coefficient values for $m$ and $k$ are given by the sum from 0 to $L-1$. Since the STFT information is a complex function, a spectrogram - a three-Dimensional (3-D) plot is used to present its data. The spectrogram mathematical expression is shown in (19); where $m$ and $k$ retain their definitions.

$$
\operatorname{10log}_{10}(|X[m ; k]|)
$$

\section{(b) Resolution Optimization}

Due to the STFT time-frequency compromise, the width of the window that determine time or frequency optimization must be carefully selected. In practice, a short window results in many time slices, a wide band spectrogram; and thus, a better time resolution. On the other hand, a long window results in more DFT points, a narrow band spectrogram; and thus, a better frequency resolution [19]. In this paper, a long window (Hanning) was selected resulting in a better frequency resolution (see Fig. 6)

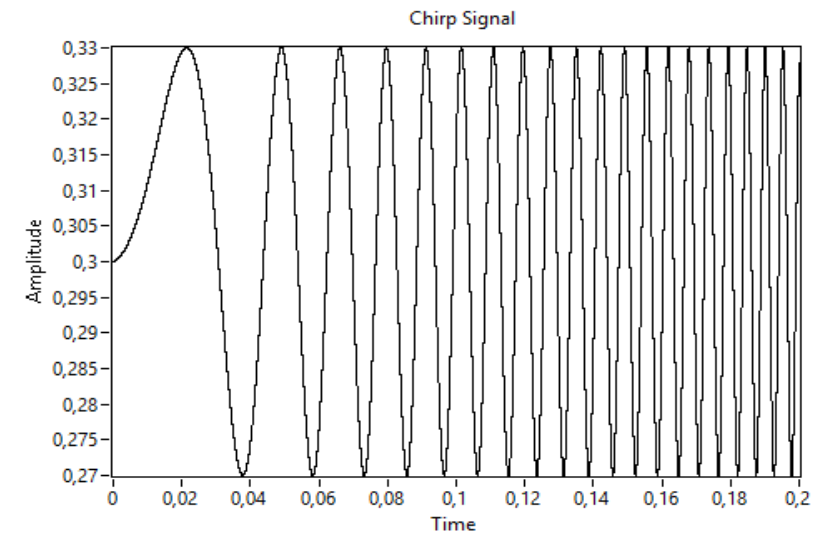

Fig. 5(a) Chirp Broadband Excitation, $\mathrm{CF}=1.4$

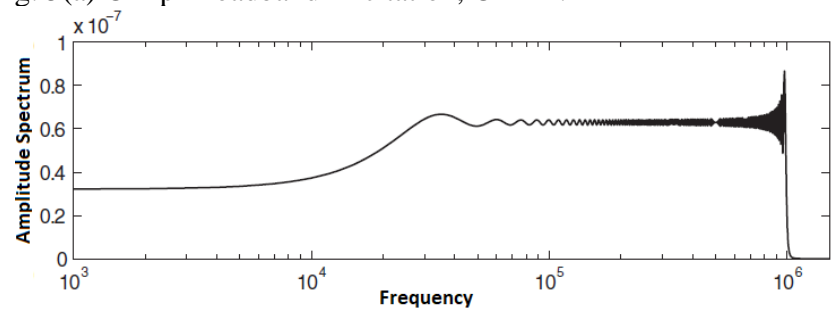

Fig. 5(b) Frequency spectrum of the chirp signal [10]

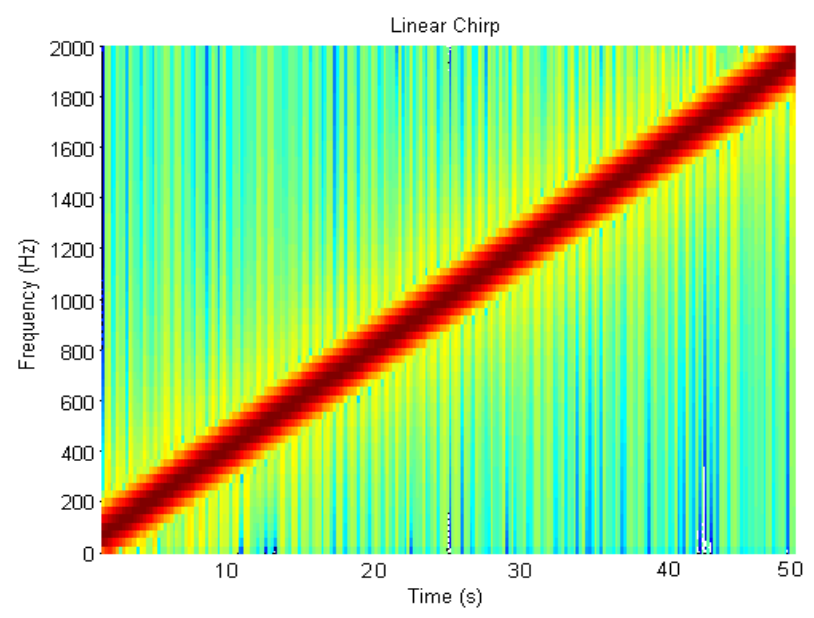

Fig. 6 STFT with a narrow band resolution

\section{ONLINE CONDITION MONITORING SYSTEM}

In an online condition monitoring system, state estimation of the battery can be accomplished while the system is in operation. In this paper, a power converter that is typically used to interface the battery with the load for current and voltage regulation functions, is utilized for online condition monitoring of the batteries through closed loop control of the power converter and duty-cycle perturbation. The major advantage derived from using a converter to carry out Electrochemical Impedance Spectroscopy measurement is that it obliterates disturbances in the system that might have occurred due to a disconnection of the system in operation or an application of an external ac signal through a signal generator [13], [20] - [24].

Online EIS was achieved on electrochemical systems in [13], [20] - [23]. In [20], online EIS was achieved using a single perturbation cycle, controlled via closed loop control of the power converter. In [21], online EIS was achieved on a lead 
acid, nickel-metal-hydride and lithium ion battery via a DCDC boost/buck converter and duty-cycle perturbation - as also applied in this paper. In [22], online condition monitoring was achieved using a custom designed switch-mode amplifier (incorporated into a battery charger) that produces perturbation current for EIS measurement. In [20] - [22], the perturbation signal was a single-sine; therefore, even though these online systems were able to achieve real-time condition monitoring, they were still constrained by the time-limitation of the classical EIS. EIS measurement time was significantly reduced in [13] and [23] using multisine broadband perturbation signals. In [13], online EIS was accomplished using a boost/buck converter. In [23], online EIS was implemented on a Proton Exchange Membrane (PEM) fuel cell. This was achieved using a power converter with binary sequence perturbation signal. Like [20] - [22], the drawback in [13] and [23], is that the broadband signals used are still constrained by the lowest frequency of the EIS perturbation signal. This paper hence aims to further reduce the measurement time associated with EIS test for two different batteries - lead acid and lithium NCM - with two different time constants. The chirp signal will be utilized to achieve this, since it can be generated in an infinitesimal time. More so, its precision at the most important frequency decade (low-mid frequencies) of the impedance spectrum of batteries (as investigated in [6]), makes it the broadband signal of choice for online EIS measurement on both batteries.

\section{A. Mode of Operation of Proposed Online Condition Monitoring System}

An online condition monitoring system as shown in Fig. 7 is proposed to undertake EIS measurement on batteries. To achieve this, a power (boost/buck) converter capable of interfacing the battery and electronic load is designed. The power converter invariably requires a control mechanism to carry out condition monitoring, while maintaining its primary function of varying the output voltage as per the associated duty cycle. Since a converter is inherently dynamic - because of the non-linear time-varying nature of the PWM and switching processes, a model that is time invariant and quasi-linear is needed to be derived [25] - [26]. In this regard, a good model representation of the converter is determined by state space averaging and small-signal analysis. The state space averaging technique is firstly utilized to eliminate the time variance in the power stage; however, this induces certain nonlinearities to the power stage average model. Therefore, linearization of the operating point of the power stage is realized using a smallsignal model. This results in a linear time-invariant small-signal model, which is converted into the frequency-domain, to provide the power stage dynamic's transfer function [25] - [26]. Following modelling of the converter, the open-loop control system is realized. The open-loop system depicts the transfer function of the inductor current and duty cycle; and the capacitor voltage and the duty cycle. Nonetheless, the openloop system has no information of the system output, which is critical in battery application due to its varying voltage.

A robust closed loop control system is consequently applied to control the DC bus voltage (as typically required in a converter) and perform EIS measurement through duty cycle perturbation. Moreover, the parameters (gain) of the control is tuned in such a way that it satisfies the control criterion for both-

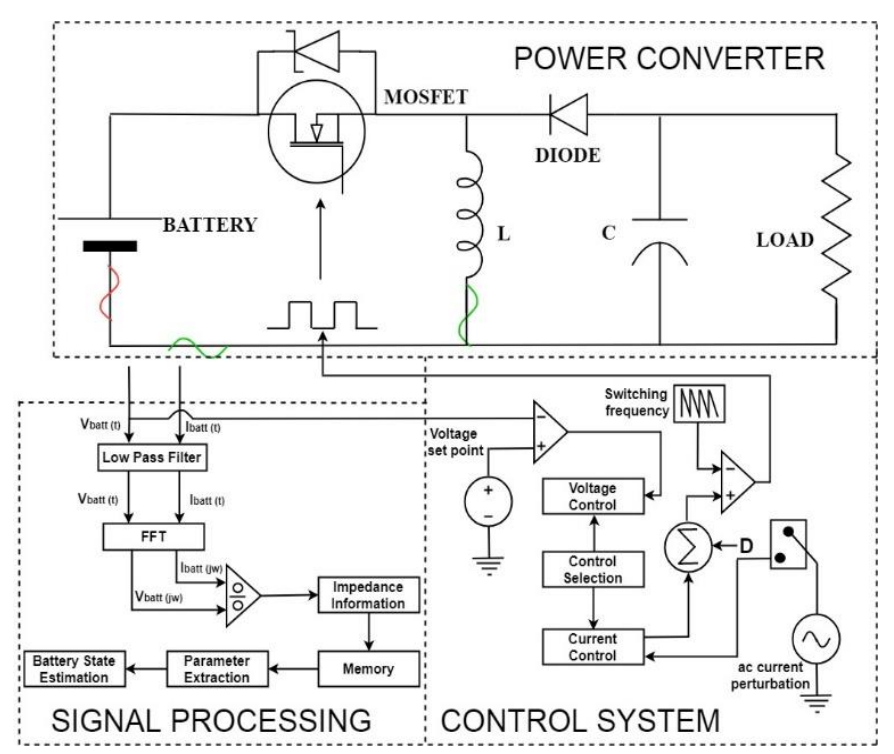

Fig. 7 Proposed online condition monitoring system.

the lithium NCM and lead acid batteries. This control switches sequentially, commencing from the DC bus voltage control and then the EIS measurement (current control). Following the derivation of the time-domain ac current and voltage, the signal is passed through a low pass-filter, so that the switching frequency components of the PWM is eliminated from the signal. The signal is then converted to the frequency domain using Fourier Transform. The frequency domain impedance information is subsequently determined using the Ohm's law relationship as briefly asserted in Section II. The equivalent circuit parameters are also extracted from the impedance information using CNLS as also already discussed in Section II.

\section{B. Control Algorithm for EIS measurement}

Open-loop control has no information on the system output and assumes that the output voltage remains constant [27]. A robust closed-loop control is implemented for not only voltage control but to ensure that the system has a high disturbance rejection (e.g. load change); because current injection (perturbation) for impedance spectroscopy measurement can only take place when the system is stable. In an online condition monitoring system, the output voltage must be constant regardless of variations in the input voltage. Since in batteries, the voltage varies as the battery gets discharged over time, the output voltage must thus be kept constant regardless of the applied load. To accomplish this, the power converter invariably requires a feedback mechanism. Hence, the converter control input - duty cycle must be continuously varied in a such a way that the output voltage is always equal to the design value [25] - [26]. Fig. 8 shows the flow chart of the control algorithm applied to the proposed online condition monitoring system.

Before EIS measurement is taken, the controller detects if the power converter is operating within the acceptable voltage set point region. Based on the stability (chemical and control) investigation carried out on both batteries by the same Authors in [5], the voltage requirements are set to be stricter for the lead acid battery $( \pm 5 \%)$ compared to that of the lithium NCM battery $( \pm 10 \%)$. Now, if the converter is not operating within the acceptable voltage region (i.e. $\mathrm{V}_{\text {batt }} \approx$ set point threshold), the- 


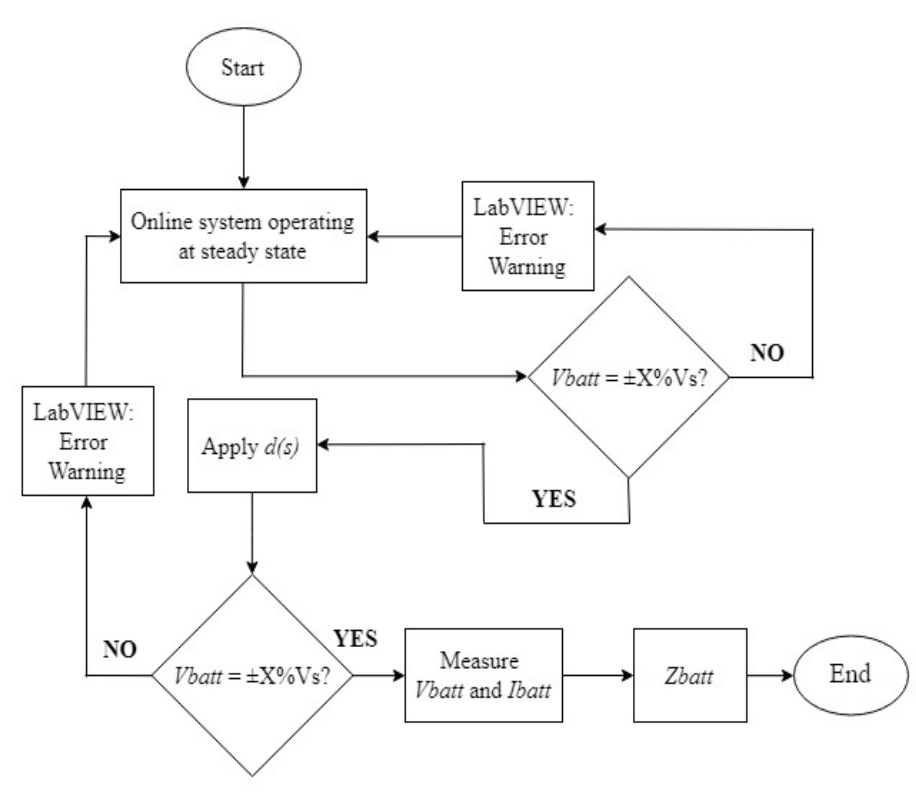

Fig. 7 Flowchart of proposed online condition monitoring control algorithm.

system generates an "error warning," and waits for $t$ seconds until the system attains steady state. If the converter is operating within the acceptable voltage region, voltage control is suspended, and duty cycle sinusoidal excitation (ac current injection) is applied for impedance spectroscopy measurement. This duty cycle excitation results in generating a small sinusoidal ripple superimposed over the battery DC voltage and DC current. Furthermore, during the period that EIS measurement is taken, the system ensures that the battery voltage remains within the set point region. If the battery voltage deviates outside this threshold, the system generates an "error warning," and the measurement is stopped until a stable condition is achieved by the controller.

\section{Control Mechanism for Online Condition Monitoring}

In this sub-section, the open loop and closed loop equation are determined. The open loop transfer function is achieved using state space average and small-signal analysis. The small signal transfer function for the inductor current $I_{L}$ and capacitor voltage $V_{c}$ are thus expressed as in (20) - (21).

$$
\begin{gathered}
G_{V C}=\frac{\widetilde{v_{C}}(s)}{\tilde{d}(s)}=\frac{-\left[\frac{\left(V_{\text {batt }}-V_{C}\right)(1-D)}{L C}\right]+\frac{s I_{L}}{C}}{s^{2}+\frac{s}{R_{\text {load }} C}+\frac{(1-D)^{2}}{L C}} \\
G_{I L}=\frac{\widetilde{\tau_{L}}(s)}{\tilde{d}(s)}=\frac{\frac{s\left(V_{\text {batt }}-V_{C}\right)}{L}+\frac{\left(V_{\text {batt }}-V_{C}\right)}{R_{\text {load }} C L}+\frac{(1-D) I_{L}}{L C}}{s^{2}+\frac{s}{R_{\text {load }} C}+\frac{(1-D)^{2}}{L C}}
\end{gathered}
$$

The closed loop equation is determined from the closed loop diagram of the online system. The closed loop equation $H$ is as shown in (22):

$H=\frac{\left(K_{p} s+K_{i}+K_{d} s^{2}\right)\left(-\left[\frac{\left(V_{\text {batt }}-V_{C}\right)(1-D)}{L C}\right]+\frac{s I_{L}}{C}\right)}{s^{3}+\frac{s^{2}}{R_{\text {load }} C}+\frac{s(1-D)^{2}}{L C}+\left(K_{p} s+K_{i}+K_{d} s^{2}\right)\left(-\left[\frac{\left(V_{\text {batt }}-V_{C}\right)(1-D)}{L C}\right]+\frac{s I_{L}}{C}\right)}$

From [27], a feedback control system is stable, if and only if, all the roots of the characteristic equation fall at the left of the complex plane's imaginary axis (negative half of the s-plane). Thus, to ensure that the poles are located at the negative half of the s-plane, the roots of the poles are all taken as " -1 ."

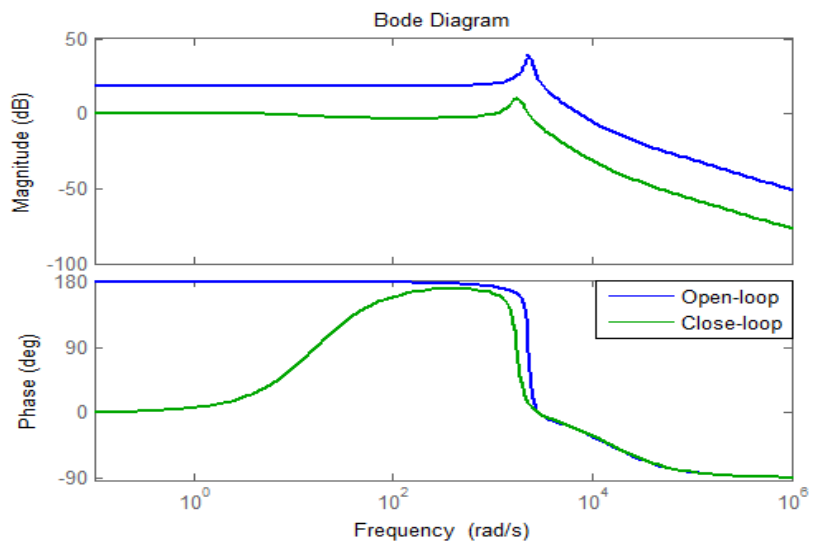

Fig. 8(b) Open and closed loop frequency response of a lead acid battery.

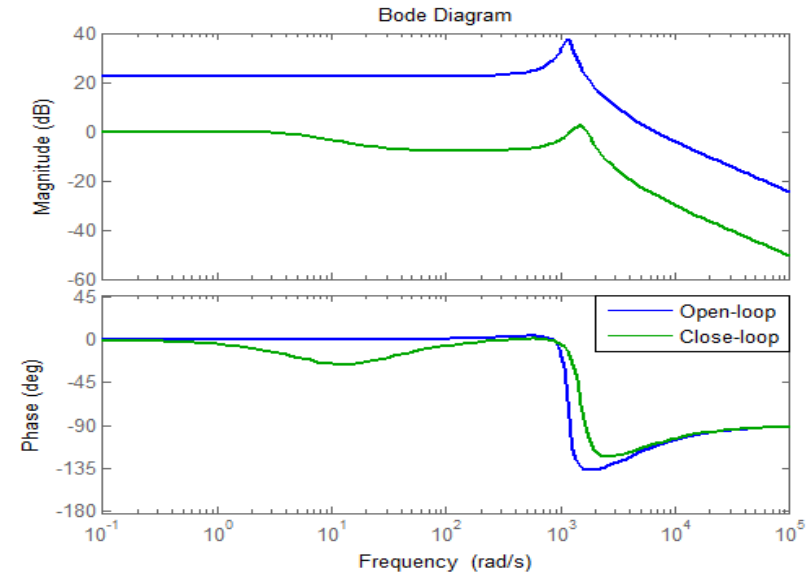

Fig. 8(a) Open and closed loop frequency response of a lithium NCM battery.

Since $(s+1)^{3}=s^{3}+3 s^{2}+3 s+1$, the coefficients are thus replaced to obtain $K_{p}, K_{i}$ and $K_{d}$. Hence, $K_{p}, K_{i}$ and $K_{d}=$ $0.05,1$ and 0 respectively. These gains result in a very robust control but suffers from control insensitivity. Consequently, the gain of the online condition monitoring system was tuned to satisfy optimal control criterion as [27]. For the lithium NCM battery the gains are tuned as: $K_{p}=0.04423$ and $K_{i}=2.214$. For the lead acid battery, the gains are tuned as: $K_{p}=$ 0.0312 and $K_{i}=1.765$. Fig. 8(a) shows the closed loop control frequency response applied to the online condition monitoring system for the lead acid battery; whereas, Fig. 8(b) shows the closed loop control frequency response applied to the online condition monitoring system for the lithium NCM battery. These gains results in a control bandwidth of about $11 \mathrm{kHz}$ for the lithium NCM battery and $12 \mathrm{kHz}$ for the lead acid battery. The closed-loop control bandwidth is fast enough to capture the frequencies of interest of both batteries and thus adequately control the system over the period of EIS measurement.

\section{Methodology AND HaRdware SETUP}

The hardware set-up is shown in Fig. 9. All measurements were undertaken at room temperature; and with the battery at a constant SoC. The batteries utilized for this experiment was a pouch shaped EIG lithium NCM 4.15V-20Ah and a cuboid shaped Pulsar sealed lead acid 12.6V-7Ah battery. Before EIS 
measurement, the batteries had previously undergone more than 30 charge-discharge cycles. The NI-DAQ controlled via the Lab VIEW software was used to estimate the impedance of the batteries. More so, the results of each test were benchmarked against an off-the-shelf EIS equipment - i.e. the Frequency Response Analyzer (FRA) controlled via the NOVA software.

The 20A booster was connected for tests were load current exceeded 2A. More so, to ensure that measurements were not undertaken in a non-linear condition, harmonic distortion analysis was applied for EIS measurement optimal perturbation amplitude determination. These corresponded to $15 \%$ of the load current for the lead acid battery and $20 \%$ of the load current for the lithium NCM battery. In all tests in this paper, the lead acid battery was operated at a $1 \mathrm{~A}$ load current - with a frequency sweep between $5 \mathrm{kHz}$ and $100 \mathrm{mHz}$. Likewise, EIS measurements were completed within: 3.4 minutes for the FRA, 10 seconds for the multisine and 5 seconds for the chirp signal. On the other hand, the lithium NCM battery was operated at a $4 \mathrm{~A}$ load current - with a frequency sweep between $2 \mathrm{kHz}$ and $20 \mathrm{mHz}$. EIS measurements were completed within: 6.8 minutes for the FRA, 50 seconds for the multisine and 25 seconds for the chirp signal. In theory, the chirp signal can be generated in an infinitesimal time - as low as, one-tenth the time of the multisine and PRBS. However, the chirp signal duration must be long enough to maximize the signal energy and thus SNR; which is why the chirp signal was designed to be generated only half the time required for processing the lowest frequency.

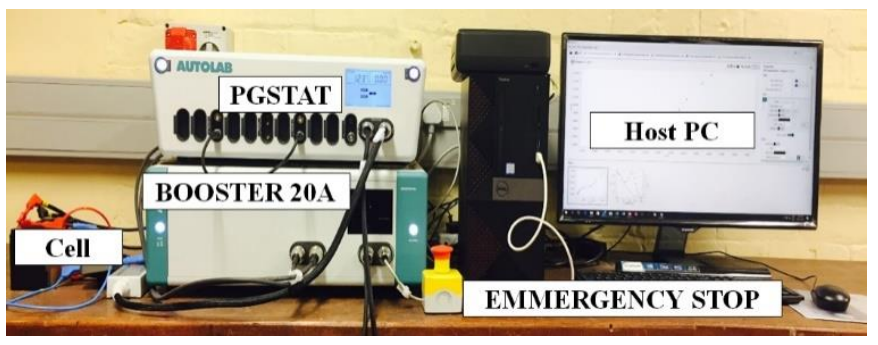

Fig. 9(a) PGSTAT set-up

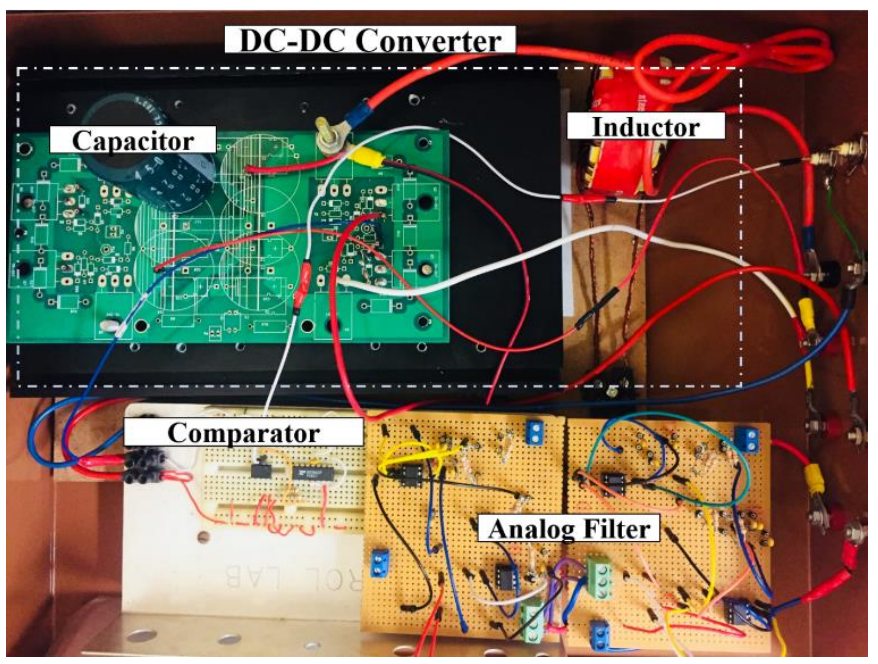

Fig. 9(b) Online condition monitoring hardware set-up

\section{RESULT AND DISCUSSIONS}

Fig. 10 shows the offline broadband EIS results compared against the FRA for both batteries. It is evident from the result that broadband signals can significantly shorten the time required for EIS measurement. In the same vein, Fig. 11 shows the results obtained from the online broadband EIS compared against the FRA for both batteries. From the results, it can be deduced that broadband EIS can be implemented when the system is in operation. As earlier discussed, the increased speed that broadband EIS offers, results in a compromise of the impedance spectrum accuracy. It is therefore important to analyse the time-frequency properties of these signals (already established in Section III); alongwith their impedance spectrum quality to ascertain the most suitable signal tool for online condition monitoring. Table 2 presents the extracted parameters of the FRA, which are used to benchmark the offline and online test. Table 3 presents the deviation of the different broadband signals (offline and online) explored in this paper against the already determined FRA extracted impedance parameters. It is evident from the extracted parameters for both the lithium NCM and lead acid (offline and online) tests that the chirp signals possesses the least deviation from the FRA at the ohmic series resistance (R1); charge transfer resistance (R2); and doublelayer capacitance (CPE). However, the chirp signal performs poorly at the diffusion region $(\mathrm{W})$. Another thing of note is the fact that the lead acid battery is more sensitive to the effect of broadband signals compared to the lithium NCM battery. This can be attributed to the difference in the electrochemistry of both batteries.

As already established, the multisine possesses the worst $\mathrm{CF}$ compared to the PRBS and chirp but shares a closer Nyquist plot with the FRA over the impedance spectrum. This can be-

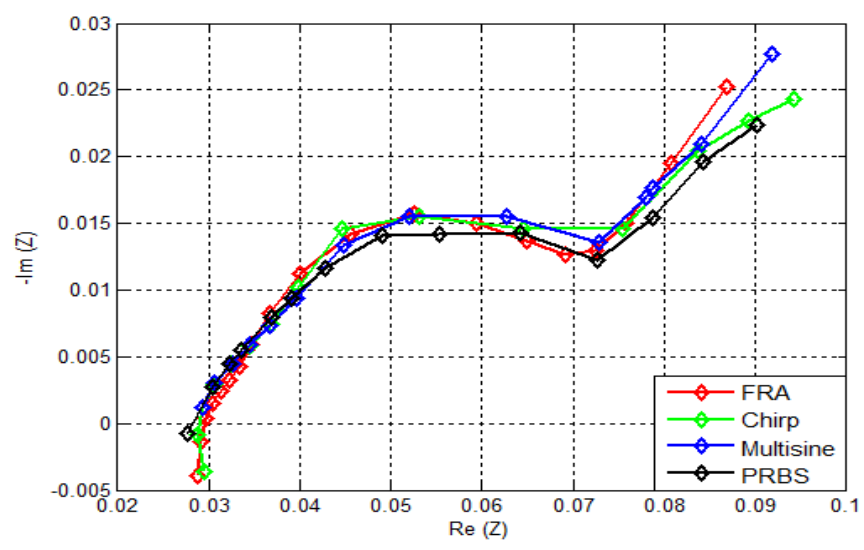

Fig. 10(a) Offline broadband impedance spectrum for lead acid

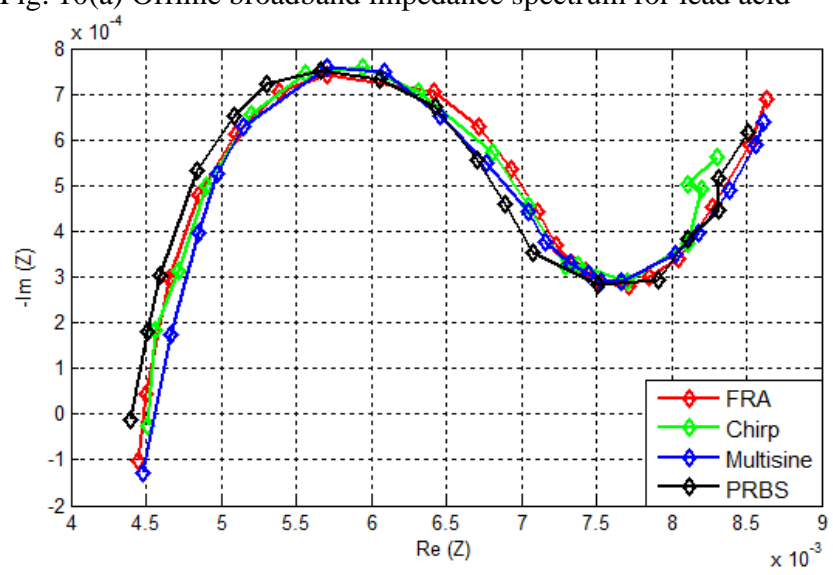

Fig. 10(b) Offline broadband impedance spectrum for lithium NCM 


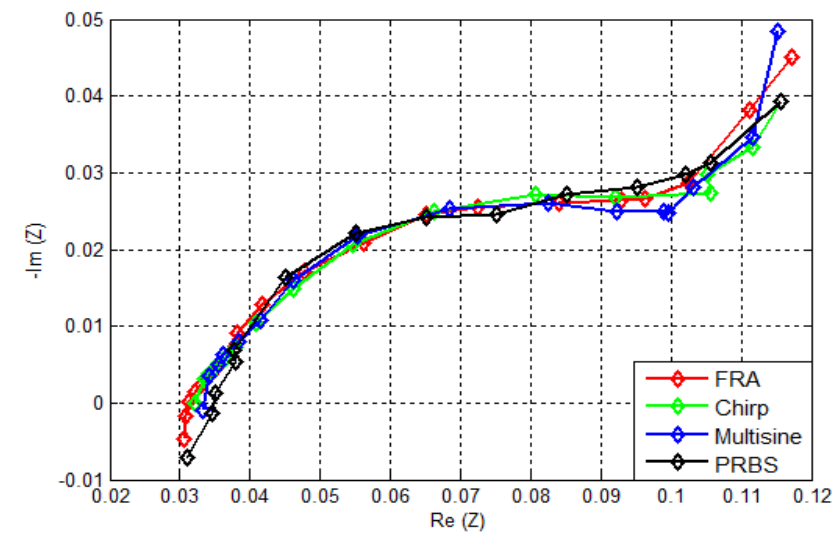

Fig. 11(a) Online broadband impedance spectrum for lead acid

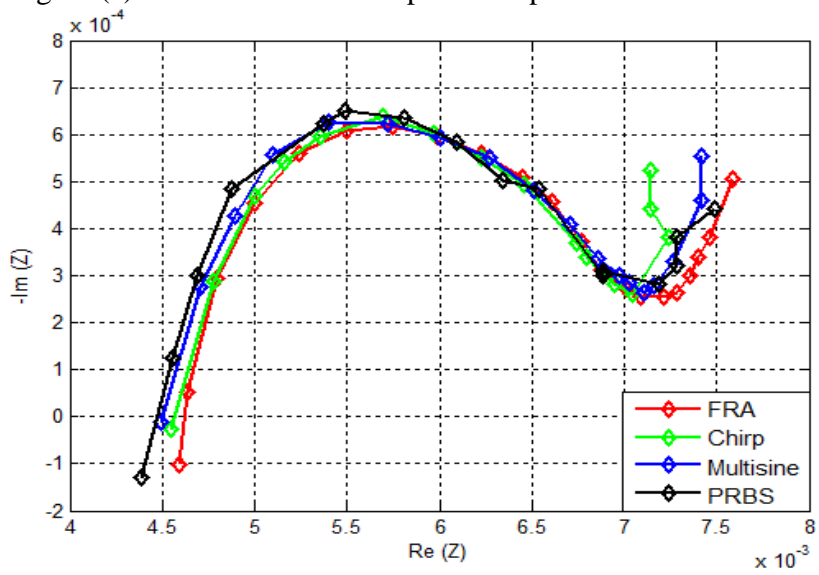

Fig. 11(b) Online broadband impedance spectrum for lithium NCM

attributed to the excitation energy of the multisine that is evenly distributed across the user-selected set of frequencies. As also already established, the chirp signal can be generated in an infinitesimal time but is designed to be generated in one- half the time required to process the lowest frequency. The chirp signal has a high SNR over the EIS measurement frequency range, because of its flat spectrum. However, most of its excitation power is concentrated at the high frequency; which implies that the high frequency decade will benefit the most from the high SNR of the chirp signal. This theoretical deduction correlated with the laboratory results, as the chirp signal performed the best at the mid-high frequency region (i.e. $\mathrm{R} 1, \mathrm{R} 2$ and CPE). Moreover, from previous work in [5], these parameters are sufficient for state estimation of both batteries.

Table 2. Parameter extracted from FRA measurement for both offline and online investigation.

\begin{tabular}{lllll}
\hline \hline \multicolumn{5}{c}{ Lead acid (FRA) } \\
\hline \hline \multirow{5}{*}{ Offline test } & R1 & R2 & CPE & W \\
Online test & 0.0294 & 0.0304 & 0.640 & 0.8321 \\
\hline \hline \multicolumn{5}{c}{ Lithium NCM (FRA) } \\
\hline \hline \multirow{5}{*}{ Offline test } & 0.004405 & 0.00305 & 13.08 & 0.8544 \\
Online test & 0.004650 & 0.00281 & 13.28 & 0.00222 \\
\hline
\end{tabular}

Table 3. Parameter deviation of offline and online broadband signals against FRA measurement

\begin{tabular}{|c|c|c|c|c|}
\hline \multicolumn{5}{|c|}{ Lead acid (Offline) } \\
\hline & $\Delta \mathrm{R} 1$ & $\Delta \mathrm{R} 2$ & $\overline{\Delta \mathrm{CPE}}$ & $\overline{\Delta \mathbf{W}}$ \\
\hline Chirp & 0.0132 & 0.0157 & 0.113 & 0.0233 \\
\hline Multisine & 0.0148 & 0.0189 & 0.119 & 0.00987 \\
\hline PRBS & 0.0267 & 0.0201 & 0.203 & 0.0259 \\
\hline \multicolumn{5}{|c|}{ Lithium NCM (Offline) } \\
\hline & $\Delta \mathrm{R} 1$ & $\Delta \mathrm{R2}$ & $\triangle \mathrm{CPE}$ & $\Delta \mathbf{W}$ \\
\hline Chirp & 0.000081 & 0.0000771 & 0.23 & 0.000143 \\
\hline Multisine & 0.0001221 & 0.0000971 & 0.44 & $9.7 \mathrm{E}-05$ \\
\hline PRBS & 0.0001319 & 0.0001431 & 0.93 & 0.000236 \\
\hline \multicolumn{5}{|c|}{ Lead acid (Online) } \\
\hline & $\Delta \mathrm{R} 1$ & $\Delta \mathrm{R2}$ & $\overline{\Delta \mathrm{CPE}}$ & $\overline{\Delta W}$ \\
\hline Chirp & 0.0158 & 0.0163 & 0.125 & 0.0269 \\
\hline Multisine & 0.0182 & 0.0195 & 0.137 & 0.00109 \\
\hline PRBS & 0.0207 & 0.0214 & 0.225 & 0.0273 \\
\hline \multicolumn{5}{|c|}{ Lithium NCM (Online) } \\
\hline & $\Delta \mathrm{R} 1$ & $\Delta \mathrm{R2}$ & $\triangle \mathrm{CPE}$ & $\Delta \mathbf{W}$ \\
\hline Chirp & 0.000092 & 0.0000854 & 0.57 & 0.000154 \\
\hline Multisine & 0.0001392 & 0.0001221 & 0.78 & 0.000113 \\
\hline PRBS & 0.0001439 & 0.0001319 & 1.03 & 0.000348 \\
\hline
\end{tabular}

Table 3. Summary of results for the different broadband signal for the lead acid and lithium NCM batteries

\begin{tabular}{lcccc}
\hline \hline \multicolumn{1}{c}{ Metrics } & FRA & Multisine & PRBS & Chirp \\
\hline \hline Measurement time & Long & Short & Short & Shorter \\
EIS accuracy & Yes & Relative & Relative & Relative \\
Online application & No & Yes & Yes & Yes \\
\hline \hline
\end{tabular}

\section{CONCLUSION}

Broadband excitation signals can be used as perturbation signals; to shorten the time required for EIS measurement, without the need to disconnect the battery from the system in operation (see summary of our findings in Table 3). However, it is important to note that the increased speed offered by broadband EIS, results in a compromise of the impedance spectrum accuracy. Therefore it is crucial to understand: the time-frequency characteristics of the different broadband signals and how it affects SNR; how the choice of the different broadband signal can influence instrumentation of the system in operation; and how the chemistry of batteries can impact the methodology of EIS measurement, the design of the broadband signal and control system. Over the critical EIS range of frequencies of both batteries, results from the multisine excitation shares the closest resemblance to the FRA, compared to the PRBS and chirp signals. Nonetheless, the chirp signal offers a shorter measurement duration - half the time required for multisine and PRBS excitation. Furthermore, the chirp 
signal accurately determines the most crucial impedance parameters required to determine the state of both batteries; compared to the PRBS and multisine. The chirp signal is thus proposed as the most suitable broadband signal for online condition monitoring of sealed lead acid and lithium NCM batteries. Given the nature of these extremely different batteries (from a time-constant and electrochemistry view point); the Author's are thus convinced that this proposed online technique is applicable to other commercially available batteries.

\section{REFERENCES}

[1] D. Klot, "Characterization and Modeling of Electrochemical Energy Conversion Systems by Impedance Techniques," Ph.D. Thesis, Karlsruher Institut Für Technologie (KIT), 2012.

[2] Ross Kerley, "Automotive Lead-Acid Battery State-of-Health Monitoring System," Master's Thesis, Virginia Polytechnic Institute and State University, 2014.

[3] Wladislaw Waag, Christian Fleischer, Dirk Uwe Sauer "Critical review of the methods for monitoring of lithium-ion batteries," Elsevier Journal of Power Sources, vol. 258, pp. 321-339, 2014.

[4] M. Berecibar, I. Gandiaga, I. Villarreal, N. Omar, J. Van Mierlo, P. Van den Bossche, "Critical review of state of health estimation methods of Liion batteries for real applications," Elsevier Renewable and Sustainable Energy Reviews 56, 572-587, 2016.

[5] Olakunle Alao, Paul Barendse, "Characterization of Lithium NCM and Sealed Lead Acid Batteries using Electrochemical Impedance Spectroscopy," IEEE Power Africa, Cape Town, 2018.

[6] Olakunle Alao, Paul Barendse, "Online Condition Monitoring of Sealed Lead Acid \& Lithium Nickel-Cobalt-Manganese Oxide Batteries using Broadband Impedance Spectroscopy," IEEE ECCE, Portland - Oregon, 2018.

[7] Evgenij Barsoukov, J. Ross Macdonald, "Impedance Spectroscopy Theory, Experiment, and Applications," Published by John Wiley \& Sons, Inc., Hoboken, New Jersey, 2005.

[8] J. J. Giner-Sanz et al. "Optimization of the Perturbation Amplitude for Impedance Measurements in a Commercial PEM Fuel Cell Using THD," 5th European PEFC \& H2 Forum, June 30 - July 3, 2015.

[9] M. Kiel, O. Bohlen, D.U. Sauer, "Harmonic analysis for identification of nonlinearities in impedance spectroscopy," Electrochimica Acta 53 pp. 7367-7374, 2008.

[10] B Sanchez et al. "Basics of broadband impedance spectroscopy measurements using periodic excitations," Institute of Physics and Engineering in Medicine, Meas. Sci. Technol. 23, 105501 (14pp), 2012.

[11] Jaan Ojarand, Mart Min \& Paul Annus, "Crest factor optimization of the multisine waveform for bioimpedance spectroscopy, Institute of Physics and Engineering in Medicine, Physiol. Meas. 35, pp 1019-1033, 2014.

[12] Benjamin Sanchez, Ramon Bragos and Gerd Vandersteen "Influence of the Multisine Excitation Amplitude Design for Biomedical Applications using Impedance Spectroscopy" 33rd Annual International Conference of the IEEE EMBS Boston, Massachusetts USA, September 3, 2011.

[13] Sean Moore, Paul Barendse, "Online Condition Monitoring of LithiumIon Batteries Using Impedance Spectroscopy," IEEE - ECCE, 2017, pp. 5617-5624.

[14] Andrej Debenjak et al., "Fast measurement of PEM fuel cell impedance based on PRBS perturbation signals and continuous wavelet transform," Journal of Power Sources 254, pp 112-118, 2014.

[15] A.J. Fairweather et al., "Battery parameter identification with Pseudo Random Binary Sequence excitation (PRBS), Journal of Power Sources 196 (2011) 9398-9406

[16] Brian Bullecks, Resmi Suresh \& Raghunathan Rengasway, "Rapid Impedance measurement using chirp signals for electrochemical system analysis," Journal of Computers \& Chemical Engineering 106, pp 421436, 2017.

[17] Min M, Land R, Paavle T, Parve T, Annus P and Trebbels D, "Broadband spectroscopy of dynamic impedances with short chirp pulses," Physiol. Meas. 32, pp. 945-958, 2011.
[18] Akindele Aroge, Paul Barendse, "Time-Frequency Analysis of the Chirp Response for Rapid Electrochemical Impedance Estimation," IEEE ECCE, Portland - Oregon, 2018.

[19] Steven W. Smith," The Scientist and Engineer's Guide to Digital Signal Processing," DSP Guide, 2nd Ed., 1999.

[20] J. A. A. Qahouq et al., "Single-Perturbation-Cycle Online Battery Impedance Spectrum Measurement Method with Closed-Loop Control of Power Converter," IEEE Transactions on Industrial Electronics, Vol. 64, No. 9, September 2017

[21] W. Huang et al. "An Online Battery Impedance Measurement Method Using DC-DC Power Converter Control," IEEE Transactions on Industrial Electronics, vol. 61, no. 11, pp. 5987-5995, 2014.

[22] R. Koch et al., "Impedance Spectroscopy for Battery Monitoring with Switched Mode Amplifiers," in 16th International Power Electronics and Motion Control Conference and Exposition, Antalya, Turkey, 2014.

[23] A. Debenjak, J. Petrovcic, P. Boskoski, B. Musizza and D. Juricic, "Fuel Cell Condition Monitoring System Based on Interconnected DC-DC Converter and Voltage Monitor," IEEE Transactions on Industrial Electronics, vol. 62, no. 8, pp. 5293-5305, 2015.

[24] Mina Abedi Varnosfaderani, Dani Strickland, "A Comparison of Online Electrochemical Spectroscopy Impedance Estimation of Batteries," IEEE Access Journal, 2018.

[25] Ned Mohan, Tore Undeland, and William Robbins, "Power Electronics Converters, Applications and Design," Jon Wiley \& Sons Inc., 2nd Ed., 1995.

[26] Charles Alexander and Mathew N.O Sadiku, "Fundamentals of Electric Circuits,", Fourth edition, The McGraw-Hill Companies, 2009.

[27] S. K. Bhattacharya, "Control Systems Engineering," Published by Pearson, $2^{\text {nd }} E d ., 2008$.

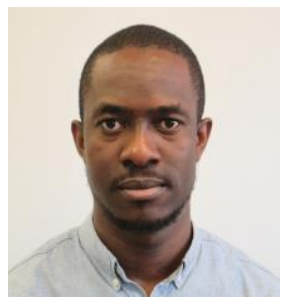

Olakunle Alao (M'15) was born in Maiduguri, Nigeria. He is currently a postgraduate student at the Advanced Machines and Energy System Research (AMES) Group, Electrical Engineering Department of the University of Cape Town, South Africa. In 2015, he completed his B.Eng. with a first-class in Electrical and Electronics Engineering from Covenant University, Nigeria. His research interest includes: power electronics for energy conversion, digital signal processing and material science for electrochemical systems. Olakunle is a member of the Engineering Council of South Africa.

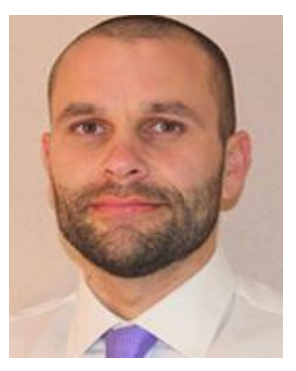

Paul Barendse (M'05) received the B.Sc., M.Sc., and Ph.D. degrees in electrical engineering from the University of Cape Town, Cape Town, South Africa, in 2001, 2005, and 2007, respectively.

In 2002, he worked for the mining company IMPLATS, where he was a Junior Engineer. He is currently with the Department of Electrical Engineering, University of Cape Town, where he is a Professor. He specializes in the areas of power electronics, drives, and machines. His research interests also include signal processing techniques applied to power applications. Prof. Barendse is a member of the South African Institute of Electrical Engineers. 